\title{
The analysis of LPI in Fujian province based on quantile regression
}

\author{
Wei Liu \\ Xiamen university of Tan Kah Kee college \\ liuwei568@xujc.com
}

\begin{abstract}
Keywords: LPI , Fujian province, quantile regression, China (Fujian) Pilot Free Trade Zone, 21st-Century Maritime Silk Road
\end{abstract}

Abstract As the China (Fujian) Pilot Free Trade Zone was set up in April 2015, the economic developed rapidly in Fujian province. While LPI has been extensively investigated, the LPI in Fujian province is relatively unexplored. This paper studies the indexes of LPI both in China and Fujian province between April 2014 and November 2016. The research focuses on the trend of LPI both in China and Fujian province, and elucidates some of the most influential indicators of LPI in Fujian province based on quantile regression. The findings of this study show that there are two possible implications for LPI in Fujian province. The first implication concerns the indexes of LPI in Fujian province which are smaller than the indexes of LPI in China. For the second one, we discuss the sub-indicators which have a significant impact on LPI in Fujian province, and the indicators include business volume, new orders, capacity utilization, average inventory and employment. In the end, we put forward some suggestions.

\section{Introduction}

Before we proceed with our discussion on the analysis of the LPI in Fujian province, we need to explain an important concept--LPI. This term has been extensively used to study a fore-indicator of economics and business conditions in China. The LPI refers to the business volume index of China logistics prosperity index, debuted in March 2013, providing an early indication of logistics activities each month. Meanwhile, The LPI in Fujian province first launched in April 2014, which contains the indexes of LPI in Fujian province. The LPI in China and the LPI in Fujian province are published by China Federation of Logistics \& Purchasing (CFLP). It often contrasts with the Logistics Performance Index (LPI) first published by the World Bank in 2007 for a sum of 150 countries.

In the last decades, the economic theorists and researchers have investigated the concept of LPI. Various groups of researchers have worked on LPI. For example, Marti ${ }^{[1,2]}$ etc have written widely about LPI. Puertas ${ }^{[3]}$ etc have discussed the importance of logistics performance in relation to European Union exports in 2005-2010. YAN and $\mathrm{WANG}^{[4]}$ have investigated the logistics performance of the Silk Road Economic Zone, which is less than the average level of the whole world. FAN and $\mathrm{YU}^{[5]}$ have done extensive work with the relationship between LPI and import and export trade. In addition, $\mathrm{Liu}^{[6]}$ has tested the periodic decomposition of China's LPI.

Most of the previous studies on LPI describe gravity equation. The studies report the problems while enhancing the logistics performance, and come up with improvement suggestions. Other studies are based on ordinary least square, which is aimed to examine the impact of logistics performance of the Belt and Road initiatives. Moreover, other studies make use of Hodrick-Prescott filter.

We did not find any publications that study the LPI in Fujian province. As China (Fujian) Pilot Free Trade Zone was set up in April 2015.There are few papers dealing with the most important indicators among all indicators of LPI in Fujian province.

The purpose of this study is to explore LPI in Fujian province through the analysis of LPI both in China and Fujian province. The specific objective in this report is to identify the trend of LPI and to elucidate some of the assumptions about the most influential indicators of LPI in Fujian province. According to the China (Fujian) Pilot Free Trade Zone establishment time, we divide the data into two parts, April 2014 to April 2015 and May 2015 to November 2016.

This paper is divided into 6 main sections. Section 1 presents the research hypothesis. Section 2 provides some background information about methodology. Section 3 proposes the data description. 
In section 4, it describes the variables and definitions. In Section 5, we present hypothesis test and result. Finally, Section 6 outlines a number of explanations for our findings and puts forward some suggestions.

\section{Hypothesis}

According to the Fujian government reports, the GDP of Fujian province grew by 8.5 percent in 2016, and Fujian province is becoming a core area of the 21st-Century Maritime Silk Road. On the other hand, the GDP of China achieved the 6.7 percent economic growth in 2016, combining these perspectives:

Hypothesis 1: The indexes of LPI in Fujian province are larger than the indexes of LPI in China.

Because the China (Fujian) Pilot Free Trade Zone was set up in April 2015, we divide the data into two parts, April 2014 to April 2015 and May 2015 to November 2016. We can promote comprehensive development of economics by pushing forward the building of the Belt and Road, so we suppose:

Hypothesis 2: The indexes of LPI in Fujian province in April 2014 and April 2015 are smaller than the indexes of LPI in Fujian province in May 2015 to November 2016.

The LPI in Fujian province contains 12 sub-indicators, different indicators lead to different results, we can get:

Hypothesis 3: The sub-indicators of the LPI in Fujian province should increase over time.

\section{Methodology}

The research is aimed at obtaining information about the relationship between the outcome LPI and the regressor sub-indicators of LPI in Fujian province. The standard linear regression is based on the conditional mean function $\mathrm{E}(\mathrm{y} \mid \mathrm{x})$. To explore the research questions, we use the method of quantile regression which is at different points in the conditional distribution of LPI in Fujian province. At the same time, we use the bootstrap method in quantile regression to estimate the coefficients, which overcomes the shortage problem of small sample.

\section{Data Description}

In this paper, the data of LPI both in China and Fujian province which covers the period from April 2014 through November 2016 is obtained from the Wind database.

On the China Federation of Logistics \& Purchasing (CFLP)'s own showing, LPI data collection took place every month. The order of presentation in LPI is as follows, questionnaires are monthly sent to over 300 logistics enterprises throughout China.

There are 12 sub-indicators in the survey, which contain cash flow, operating profit, operating cost, business volume, business expectations, new orders, logistics service charges, capacity utilization, average inventory, inventory turnover, investment in fixed asset and employment. The LPI reflects the situation of the logistics operation both in China and Fujian province. An index above 50 means an overall positive change, on the other hand, an index below 50 implies an negative change. Table 1 presents the indexes names and abbreviation of LPI in China. Moreover, the abbreviation of index and sub-indicators of LPI in Fujian province add subscript 1 respectively.

Table1 indexes name and abbreviation of LPI in China

\begin{tabular}{|c|c|}
\hline Abbreviation & Indexes name \\
\hline lp & China logistics prosperity index \\
\hline cf & cash flow \\
\hline op & operating profit \\
\hline oc & operating cost \\
\hline bv & business volume \\
\hline be & business expectations \\
\hline no & new orders \\
\hline
\end{tabular}




\begin{tabular}{|c|c|}
\hline sc & logistics service charges \\
\hline $\mathrm{cu}$ & capacity utilization \\
\hline $\mathrm{ai}$ & average inventory \\
\hline it & inventory turnover \\
\hline $\mathrm{fa}$ & investment in fixed asset \\
\hline $\mathrm{em}$ & employment \\
\hline
\end{tabular}

Cash flow reflects the important index changes in logistics enterprise in working capital turnover. Operating profit is an important index of logistics cost variation. Operating cost is the main index showing the changes of logistics efficiency. Business volume indicates logistics business activities activity. Business expectations illustrates the prospect of industry development by experts. New orders reflect the change of demand in logistics industry. Logistics service charges shows logistics industry changes in prices of Foreign Service charge. Capacity utilization demonstrates the degree of change in the facility utilization in related business activities. Average inventory displays the change of the quantity of the goods and the storage security of customers. Inventory turnover presents the changes of logistics enterprise working capital's turnover. Investment in fixed asset shows the new change of the fixed assets investment. Employment manifests the demand of employee.

The LPI in China overall descriptive statistics of each index is shown in Table 2. Table 2 shows the number of each index $(\mathrm{N})$, mean value(mean), maximal value(max), minimal value(min), 25th percentile(p25), 50th percentile(p50), 75th percentile(p75), standard deviation(sd) and variance(var).

Table 2 Descriptive statistics of each index of LPI in China

\begin{tabular}{|c|c|c|c|c|c|c|c|c|c|}
\hline index & $\mathrm{N}$ & mean & $\max$ & $\min$ & $\mathrm{p} 25$ & $\mathrm{p} 50$ & $\mathrm{p} 75$ & sd & var \\
\hline lp & 32 & 55.4 & 59.3 & 50.0 & 54.2 & 55.1 & 57.2 & 2.3 & 5.4 \\
\hline cf & 32 & 50.6 & 56.6 & 45.9 & 48.5 & 50.8 & 52.1 & 2.3 & 5.4 \\
\hline op & 32 & 49.4 & 53.9 & 43.2 & 48.8 & 49.8 & 51.0 & 2.6 & 6.5 \\
\hline oc & 32 & 55.0 & 61.4 & 50.7 & 53.0 & 54.9 & 56.8 & 2.3 & 5.3 \\
\hline bv & 32 & 55.4 & 59.3 & 50.0 & 54.2 & 55.1 & 57.2 & 2.3 & 5.4 \\
\hline be & 32 & 58.5 & 66.4 & 51.2 & 54.8 & 59.4 & 61.9 & 4.2 & 17.9 \\
\hline no & 32 & 54.7 & 60.1 & 50.7 & 53.4 & 54.2 & 56.1 & 2.2 & 4.9 \\
\hline sc & 32 & 49.9 & 52.3 & 47.7 & 49.3 & 49.6 & 50.7 & 1.1 & 1.1 \\
\hline cu & 32 & 54.2 & 58.7 & 48.8 & 52.1 & 54.6 & 56.5 & 2.9 & 8.1 \\
\hline ai & 32 & 52.4 & 58.8 & 44.4 & 50.3 & 52.5 & 54.6 & 3.0 & 8.9 \\
\hline it & 32 & 52.6 & 58.6 & 48.2 & 49.8 & 52.3 & 54.6 & 3.2 & 10.2 \\
\hline fa & 32 & 53.2 & 61.1 & 47.3 & 50.7 & 52.9 & 54.3 & 3.3 & 10.8 \\
\hline em & 32 & 51.2 & 56.9 & 44.4 & 49.9 & 51.2 & 52.5 & 2.3 & 5.5 \\
\hline
\end{tabular}

As is shown in Table 2, during April 2014 and November 2016, the mean value of China logistics prosperity index(lp) is 55.4, well above the critical 50-mark, Moreover, the mean values of indexes in China are almost larger than 50, except operating profit(op) and logistics service charges which are 49.4 and 49.9 respectively. The minimum of China logistics prosperity index(lp) is 50.0 , the maximum of China Logistics prosperity index(lp) is 59.3, but China logistics prosperity index(lp), operating cost(oc), business volume(bv), business expectations(be) and new orders(no) exceed 50.

The percentile results are given in Table2. We can find that the indexes in 25th less than 50 includes cash flow(cf), operating profit(op), service charges(sc), capacity utilization(cu), average inventory(ai), inventory turnover(it), investment in fixed asset(fa) and employment(em). At the same time, the indexes in 50th percentile and in 75th percentile are above 50 fully.

Table 3 Descriptive statistics of each index in Fujian province

\begin{tabular}{|c|c|c|c|c|c|c|c|c|c|}
\hline stats & $\mathrm{N}$ & mean & $\max$ & $\min$ & $\mathrm{p} 25$ & $\mathrm{p} 50$ & $\mathrm{p} 75$ & $\mathrm{sd}$ & var \\
\hline lp1 & 32 & 54.3 & 57.3 & 50.6 & 53.5 & 54.4 & 55.2 & 1.5 & 2.3 \\
\hline cf1 & 32 & 54.2 & 58.4 & 48.2 & 52.7 & 54.4 & 55.9 & 2.6 & 6.6 \\
\hline op1 & 32 & 51.2 & 59.2 & 43.6 & 48.1 & 51.9 & 53.8 & 4.2 & 17.7 \\
\hline oc1 & 32 & 59.5 & 66.5 & 53.4 & 56.6 & 60.2 & 62.0 & 3.8 & 14.3 \\
\hline
\end{tabular}




\begin{tabular}{|c|c|c|c|c|c|c|c|c|c|}
\hline bv1 & 32 & 55.7 & 60.7 & 49.2 & 54.6 & 55.5 & 57.6 & 2.4 & 5.8 \\
\hline be1 & 32 & 56.6 & 61.7 & 49.0 & 54.0 & 56.2 & 60.0 & 3.5 & 12.2 \\
\hline no1 & 32 & 54.2 & 59.0 & 49.1 & 52.5 & 53.6 & 55.3 & 2.5 & 6.1 \\
\hline sc1 & 32 & 53.7 & 59.0 & 45.6 & 51.6 & 54.0 & 55.5 & 2.8 & 8.1 \\
\hline cu1 & 32 & 55.6 & 60.9 & 45.5 & 54.8 & 55.7 & 56.8 & 2.4 & 5.8 \\
\hline ai1 & 32 & 49.1 & 56.6 & 44.3 & 47.4 & 48.2 & 50.8 & 2.9 & 8.7 \\
\hline it1 & 32 & 51.6 & 56.6 & 43.7 & 50.3 & 51.7 & 53.0 & 2.5 & 6.4 \\
\hline fa1 & 32 & 41.3 & 47.4 & 35.9 & 39.8 & 40.5 & 43.5 & 2.8 & 8.1 \\
\hline em1 & 32 & 53.1 & 55.9 & 50.0 & 51.7 & 53.2 & 54.1 & 1.6 & 2.6 \\
\hline
\end{tabular}

It is can be seen in Table 3, during April 2014 and November 2016, the mean values of the all index in Fujian province are almost more than 50, except average inventory in Fujian province (ai1) and investment in fixed asset in Fujian province (fa1) which are 49.1 and 41.3 respectively. The minimum of the all indexes is less than 50 in Fujian province. However, LPI in Fujian province (lp1), operating cost in Fujian province (oc1), employment in Fujian province (em1) are more than 50. Specially, investment in fixed asset in Fujian province (fa1) is 35.9, which is far below 50.

The results of percentile are shown in Table 3 . We can notice that the indexes in 25 th percentile not exceed 50, which includes operating profit in Fujian province (op1), average inventory in Fujian province (ai1), investment in fixed asset in Fujian province (fa1). Meanwhile, the indexes in 50th percentile and in 75th percentile are above 50 fully, except average inventory in Fujian province (ai1) and investment in fixed asset in Fujian province (fa1).

As it is clearly demonstrated in table 2 and Table 3, The means of each index of China logistics prosperity index(lp) is larger than the means of each index of LPI in Fujian province (lp1), which contains China logistics prosperity index(lp), business expectations(be), new orders(no), average inventory(ai), inventory turnover(it), investment in fixed asset(fa) and employment(em), rejecting the hypothesis 1 test.

Table 4 Descriptive statistics of each index in Fujian province between Apr.2014 and Apr. 2015

\begin{tabular}{|c|c|c|c|c|c|c|c|c|c|}
\hline stats & $\mathrm{N}$ & mean & $\max$ & $\min$ & $\mathrm{p} 25$ & $\mathrm{p} 50$ & $\mathrm{p} 75$ & $\mathrm{sd}$ & var \\
\hline lp1 & 13 & 55.0 & 57.3 & 51.4 & 54.2 & 55.0 & 55.7 & 1.6 & 2.4 \\
\hline cf1 & 13 & 53.7 & 57.2 & 49.5 & 52.0 & 53.8 & 55.4 & 2.3 & 5.2 \\
\hline op1 & 13 & 52.2 & 59.2 & 43.7 & 49.5 & 52.4 & 57.4 & 5.1 & 26.5 \\
\hline oc1 & 13 & 61.5 & 64.9 & 56.6 & 60.8 & 61.2 & 63.0 & 2.2 & 4.9 \\
\hline bv1 & 13 & 57.4 & 60.7 & 54.7 & 56.0 & 57.8 & 58.3 & 1.8 & 3.3 \\
\hline be1 & 13 & 58.1 & 61.4 & 55.1 & 56.4 & 57.9 & 60.2 & 2.2 & 4.6 \\
\hline no1 & 13 & 55.4 & 58.9 & 52.3 & 53.1 & 55.1 & 57.7 & 2.4 & 5.8 \\
\hline sc1 & 13 & 52.5 & 58.8 & 45.6 & 50.6 & 51.8 & 53.8 & 3.6 & 12.9 \\
\hline cu1 & 13 & 55.5 & 60.9 & 45.5 & 55.2 & 56.2 & 57.1 & 3.5 & 12.5 \\
\hline ai1 & 13 & 49.2 & 56.6 & 44.3 & 46.5 & 48.3 & 50.9 & 3.7 & 13.7 \\
\hline it1 & 13 & 52.9 & 56.6 & 43.7 & 52.0 & 53.1 & 54.8 & 3.4 & 11.6 \\
\hline fa1 & 13 & 43.0 & 47.4 & 40.2 & 41.0 & 41.9 & 44.4 & 2.5 & 6.2 \\
\hline em1 & 13 & 52.4 & 55.3 & 50.0 & 51.4 & 51.7 & 53.0 & 1.6 & 2.5 \\
\hline
\end{tabular}

As is shown in Table 4, during April 2014 and April 2015, The means of indexes in Fujian province are almost over 50, except for average inventory in Fujian province (ai1) and investment in fixed asset in Fujian province (fa1). The sum of the minimum indexes which is smaller than 50 in Fujian province is 7. We can compare with table 3, during April 2014 and November 2016, it's an obvious deduction that the indexes during April 2014 and April 2015 are better than the indexes during April 2014 and November 2016.

The results of percentile are provided in Table 4 . We can find that the indexes in 25th percentile does not exceed 50 which include incorporates operating profit in Fujian province(op1), average inventory in Fujian province (ai1), investment in fixed asset in Fujian province (fa1). The results are the same as the indexes in Table 4. On the other hand, the indexes in 50th percentile and in 75 th 
percentile are above 50 fully, except average inventory in Fujian province (ai1) and investment in fixed asset in Fujian province (fa1), which are the same as the indexes in Table 4.

Table 5 Descriptive statistics of each index in Fujian province between May 2015 and Nov. 2016

\begin{tabular}{|c|c|c|c|c|c|c|c|c|c|}
\hline stats & $\mathrm{N}$ & mean & $\max$ & $\min$ & $\mathrm{p} 25$ & $\mathrm{p} 50$ & $\mathrm{p} 75$ & $\mathrm{sd}$ & var \\
\hline lp1 & 19 & 53.8 & 56.4 & 50.6 & 52.9 & 53.8 & 54.8 & 1.3 & 1.8 \\
\hline cf1 & 19 & 54.6 & 58.4 & 48.2 & 52.7 & 54.7 & 57.2 & 2.8 & 7.6 \\
\hline op1 & 19 & 50.6 & 54.7 & 43.6 & 48.0 & 51.3 & 53.4 & 3.4 & 11.8 \\
\hline oc1 & 19 & 58.2 & 66.5 & 53.4 & 54.6 & 57.3 & 60.5 & 4.1 & 16.5 \\
\hline bv1 & 19 & 54.5 & 58.4 & 49.2 & 53.3 & 55.0 & 55.8 & 2.1 & 4.3 \\
\hline be1 & 19 & 55.6 & 61.7 & 49.0 & 53.5 & 54.7 & 59.8 & 3.9 & 15.3 \\
\hline no1 & 19 & 53.3 & 59.0 & 49.1 & 52.0 & 53.1 & 54.7 & 2.2 & 4.8 \\
\hline sc1 & 19 & 54.5 & 59.0 & 51.3 & 53.1 & 54.7 & 55.5 & 1.9 & 3.6 \\
\hline cu1 & 19 & 55.7 & 58.5 & 53.0 & 54.7 & 55.7 & 56.5 & 1.3 & 1.7 \\
\hline ai1 & 19 & 49.0 & 55.2 & 44.3 & 47.4 & 48.1 & 50.6 & 2.4 & 5.9 \\
\hline it1 & 19 & 50.8 & 53.0 & 48.2 & 50.1 & 50.9 & 51.7 & 1.2 & 1.4 \\
\hline fa1 & 19 & 40.2 & 46.0 & 35.9 & 39.1 & 40.1 & 40.5 & 2.6 & 6.6 \\
\hline em1 & 19 & 53.6 & 55.9 & 50.4 & 53.0 & 53.7 & 54.2 & 1.5 & 2.2 \\
\hline
\end{tabular}

As is shown in Table 5, during May 2015 and November 2016, the mean values of indexes in Fujian province are almost larger than 50, except average inventory in Fujian province (ai1) and investment in fixed asset in Fujian province (fa1) which are 49.0 and 40.2 separately. The minimum of the indexes is almost smaller than 50 in Fujian province. However, LPI in Fujian province (lp1), operating cost in Fujian province (oc1), logistics service charges in Fujian province (sc1), capacity utilization in Fujian province (cu1) and employment in Fujian province (em1) are more than 50.

The results of percentile are illustrated in Table 5. We can get that the indexes in 25th percentile is over 50 which includes LPI in Fujian province(lp1), cash flow in Fujian province(cf1), operating cost in Fujian province(oc1), business volume in Fujian province(bv1), business expectations in Fujian province(be1), new orders in Fujian province(no1), logistics service charges in Fujian province(sc1), capacity utilization in Fujian province(cu1), inventory turnover in Fujian province(it1) and employment in Fujian province(em1). Meanwhile, the indexes in 50th percentile are almost above 50, except average inventory in Fujian province (ai1) and investment in fixed asset in Fujian province (fa1), and the indexes in 75th percentile are above 50, except investment in fixed asset in Fujian province (fa1), rejecting the hypothesis 2 test.

Table 6 Pearson's correlation of the indexes in Fujian province

\begin{tabular}{|c|l|l|l|l|l|l|l|l|l|l|l|l|l|}
\hline & lp1 & cf1 & op1 & oc1 & bv1 & be1 & no1 & sc1 & cu1 & ai1 & it1 & fa1 & em1 \\
\hline lp1 & 1 & & & & & & & & & & & & \\
\hline cf1 & $0.4^{* *}$ & 1 & & & & & & & & & & & \\
\hline op1 & $0.7^{* * *}$ & $0.4^{* *}$ & 1 & & & & & & & & & & \\
\hline oc1 & $0.6^{* * *}$ & $0.4^{* *}$ & $0.5^{* * *}$ & 1 & & & & & & & & & \\
\hline bv1 & $0.9^{* * *}$ & $0.3^{*}$ & $0.6^{* * *}$ & $0.7^{* * *}$ & 1 & & & & & & & & \\
\hline be1 & 0.3 & 0.1 & 0.3 & $0.6^{* * *}$ & $0.4^{* *}$ & 1 & & & & & & & \\
\hline no1 & $0.8^{* * *}$ & 0.1 & $0.5^{* * *}$ & $0.5^{* * *}$ & $0.8^{* * *}$ & $0.3^{*}$ & 1 & & & & & & \\
\hline sc1 & -0.1 & 0.0 & 0.3 & -0.1 & -0.2 & 0.2 & -0.01 & 1 & & & & & \\
\hline cu1 & $0.6^{* * *}$ & $0.4^{* *}$ & $0.4^{* *}$ & 0.2 & $0.4^{* *}$ & -0.01 & 0.2 & -0.3 & 1 & & & & \\
\hline ai1 & $0.5^{* * *}$ & 0.2 & $0.7^{* * *}$ & $0.5^{* * *}$ & $0.4^{* * *}$ & 0.3 & $0.5^{* * *}$ & 0.3 & 0.02 & 1 & & & \\
\hline it1 & $0.7^{* * *}$ & $0.4^{* *}$ & $0.5^{* * *}$ & $0.4^{* *}$ & $0.6^{* * *}$ & 0.3 & $0.4^{* *}$ & -0.3 & $0.5^{* * *}$ & $0.4^{* *}$ & 1 & & \\
\hline fa1 & $0.6^{* * *}$ & $0.4^{* *}$ & $0.4^{* *}$ & $0.8^{* * *}$ & $0.72^{* * *}$ & $0.4^{* *}$ & $0.5^{* * *}$ & -0.1 & 0.2 & $0.5^{* * *}$ & $0.6^{* * *}$ & 1 & \\
\hline em1 & 0.08 & 0.2 & 0.06 & -0.3 & -0.2 & $-0.4^{* *}$ & $-0.3^{*}$ & 0.1 & $0.3^{*}$ & 0.1 & 0.01 & -0.1 & 1 \\
\hline
\end{tabular}

Standard errors in parentheses ${ }^{*} p<0.05,{ }^{* *} p<0.01,{ }^{* * *} p<0.001$

Table 6 summarizes the correlation coefficients between each index. We can find that LPI in Fujian province(lp1) has a positive correlation with cash flow in Fujian province(cf1), operating profit in Fujian province(op1), operating cost in Fujian province(oc1), business volume in Fujian prov- 
ince(bv1), business expectations in Fujian province(be1), new orders in Fujian province(no1), capacity utilization in Fujian province(cu1), average inventory in Fujian province(ai1), inventory turnover in Fujian province(it1), investment in fixed asset in Fujian province(fa1) and employment in Fujian province(em1), and LPI in Fujian province(lp1) has a negative effect on logistics service charges in Fujian province(sc1).

As is shown in Table 6, results of the Pearson's correlation analyses reveal that the standard errors in parentheses $p<0.001$ between LPI in Fujian province(lp1) and sub-indicators in Fujian province, which include operating profit in Fujian province(op1), operating cost in Fujian province(oc1), business volume in Fujian province(bv1), new orders in Fujian province(no1), capacity utilization in Fujian province(cu1), average inventory in Fujian province(ai1), inventory turnover in Fujian province(it1) and investment in fixed asset in Fujian province(fa1); Standard errors in parentheses $p<0.01$ is between LPI in Fujian province(lp1) and cash flow in Fujian province(cf1).

The plot shown in Figure 1 is the empirical analysis of China logistics prosperity index in Fujian province $\left(\mathrm{q}_{0.1}=53, \mathrm{q}_{0.25}=54, \mathrm{q}_{0.5}=55, \mathrm{q}_{0.75}=56\right.$ and $\left.\mathrm{q}_{0.9}=57\right)$. The distribution appears to be reasonably symmetric, at least for $0.05<\mathrm{q}<0.95$.

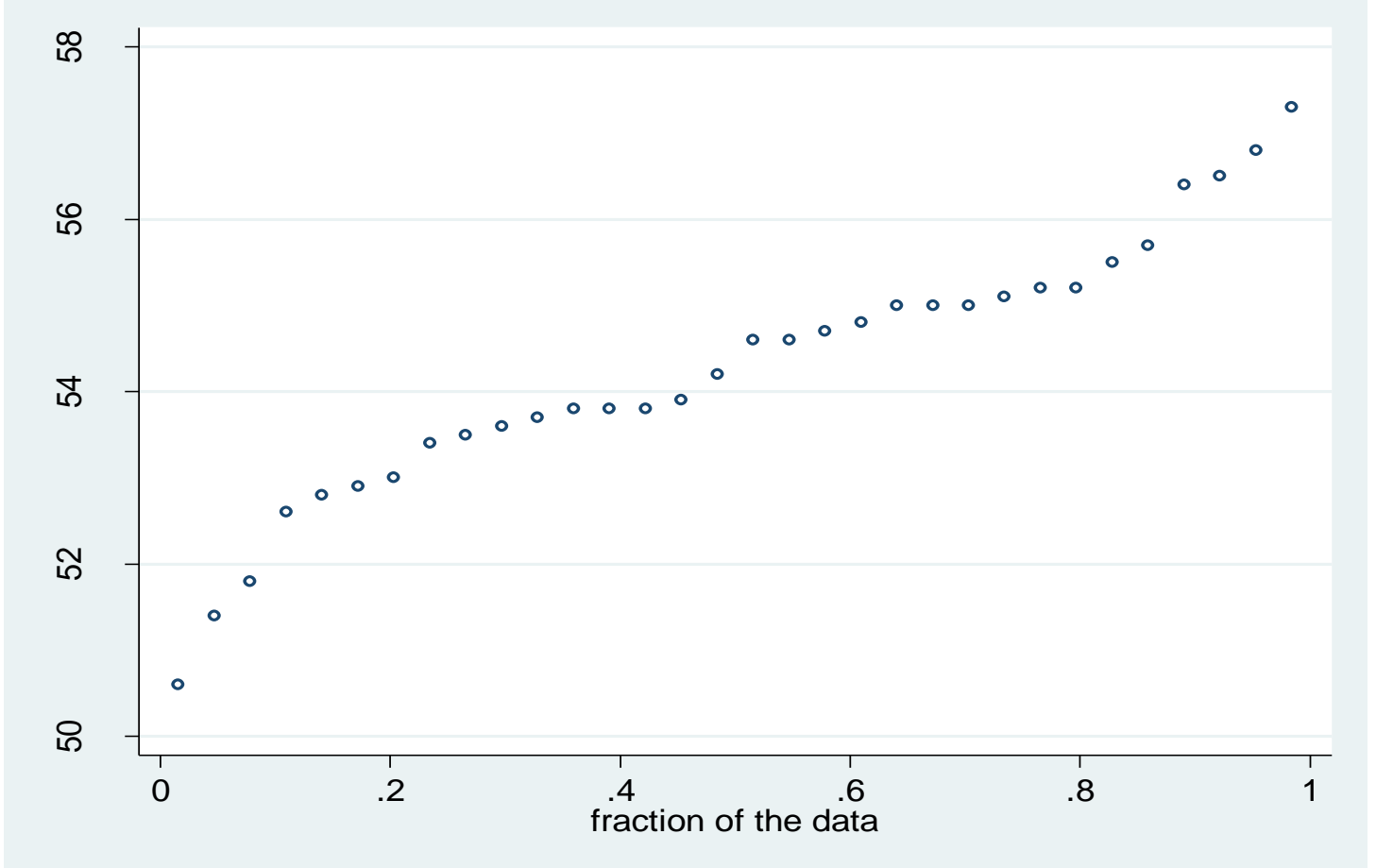

Figure 1 Quantiles of the dependent variable

\section{Results}

With respect to the relationship between LPI in Fujian province and the sub-indicators, all of them are depicted in Table 7.

Table 7 Quantiles Regression analysis of LPI in Fujian province

\begin{tabular}{ccccccc}
\hline & $(1)$ & $(2)$ & $(3)$ & $(4)$ & $(5)$ & $(6)$ \\
& ols & QR_10 & QR_25 & QR_50 & QR_75 & QR_90 \\
\hline cf1 & 0.00645 & -0.00359 & -0.00115 & 0.00179 & 0.00276 & 0.00385 \\
& $(0.0104)$ & $(0.0301)$ & $(0.0230)$ & $(0.0156)$ & $(0.0105)$ & $(0.0208)$ \\
op1 & 0.00802 & 0.000297 & 0.00377 & -0.000232 & 0.00626 & 0.00983 \\
& $(0.00828)$ & $(0.0223)$ & $(0.0155)$ & $(0.0191)$ & $(0.0124)$ & $(0.0129)$ \\
oc1 & 0.00421 & 0.0110 & 0.0104 & -0.00137 & 0.00592 & 0.00646 \\
& $(0.00902)$ & $(0.0197)$ & $(0.0216)$ & $(0.0234)$ & $(0.0165)$ & $(0.0218)$ \\
bv1 & $0.210^{* * *}$ & $0.206^{* * *}$ & $0.213^{* * *}$ & $0.244^{* * *}$ & $0.241^{* * *}$ & $0.246^{* * *}$ \\
& $(0.0265)$ & $(0.0584)$ & $(0.0657)$ & $(0.0313)$ & $(0.0512)$ & $(0.0561)$ \\
be1 & 0.00429 & -0.00683 & -0.00635 & 0.00320 & -0.00148 & -0.00302
\end{tabular}




\begin{tabular}{ccccccc} 
& $(0.00914)$ & $(0.0187)$ & $(0.0161)$ & $(0.0212)$ & $(0.0153)$ & $(0.0226)$ \\
no1 & $0.307^{* * * *}$ & $0.311^{* * *}$ & $0.311^{* * *}$ & $0.302^{* * *}$ & $0.307^{* * *}$ & $0.303^{* * *}$ \\
& $(0.0193)$ & $(0.0343)$ & $(0.0430)$ & $(0.0306)$ & $(0.0295)$ & $(0.0397)$ \\
sc1 & 0.0174 & 0.0422 & 0.0294 & 0.00156 & -0.00290 & -0.00556 \\
& $(0.0152)$ & $(0.0342)$ & $(0.0300)$ & $(0.0268)$ & $(0.0206)$ & $(0.0241)$ \\
cu1 & $0.166^{* * *}$ & $0.178^{* * *}$ & $0.169^{* * *}$ & $0.156^{* * *}$ & $0.133^{* * *}$ & $0.125^{* * *}$ \\
& $(0.0137)$ & $(0.0354)$ & $(0.0299)$ & $(0.0369)$ & $(0.0295)$ & $(0.0330)$ \\
ai1 & -0.0158 & -0.0193 & -0.0115 & -0.000748 & -0.00821 & -0.0107 \\
& $(0.0134)$ & $(0.0335)$ & $(0.0271)$ & $(0.0275)$ & $(0.0254)$ & $(0.0226)$ \\
it1 & $0.0751^{* * *}$ & $0.0769^{* *}$ & $0.0803^{* *}$ & $0.0983^{* * *}$ & $0.0992^{* * *}$ & $0.0938^{*}$ \\
& $(0.0147)$ & $(0.0348)$ & $(0.0316)$ & $(0.0260)$ & $(0.0244)$ & $(0.0452)$ \\
fa1 & $0.0224^{*}$ & 0.0354 & 0.0210 & 0.00411 & -0.00945 & -0.00520 \\
& $(0.0129)$ & $(0.0377)$ & $(0.0221)$ & $(0.0265)$ & $(0.0177)$ & $(0.0175)$ \\
em1 & $0.213^{* * *}$ & $0.205^{* * *}$ & $0.206^{* * *}$ & $0.207^{* * *}$ & $0.209^{* * *}$ & $0.208^{* * * *}$ \\
& $(0.0140)$ & $(0.0432)$ & $(0.0305)$ & $(0.0267)$ & $(0.0219)$ & $(0.0267)$ \\
ccons & -0.799 & -1.754 & -1.310 & -0.836 & 0.877 & 1.517 \\
& $(1.143)$ & $(2.088)$ & $(1.872)$ & $(3.087)$ & $(1.509)$ & $(2.955)$ \\
\hline$N$ & 32 & 32 & 32 & 32 & 32 & 32 \\
$R^{2}$ & 0.997 & & & & & \\
\hline
\end{tabular}

Standard errors in parentheses ${ }^{*} p<0.1,{ }^{* *} p<0.05,{ }^{* * *} p<0.01$

Table 7 above shows the coefficient and T statistics of sub-indicators of LPI in Fujian province.

Column(1) in Table 7 presents results for the ordinary least square regression of cash flow in $\mathrm{Fu}-$ jian province(cf1), operating profit in Fujian province(op1), operating cost in Fujian province(oc1), business volume in Fujian province(bv1), business expectations in Fujian province(be1), new orders in Fujian province(no1), logistics service charges in Fujian province(sc1), capacity utilization in Fujian province(cu1), average inventory in Fujian province(ai1), inventory turnover in Fujian province(it1), investment in fixed asset in Fujian province(fa1) and employment in Fujian province(em1), and the estimates of sub-indicators of LPI in Fujian in the column (1) are statistically significantly different from zero at the $1 \%$ level, which include business volume in Fujian province(bv1), new orders in Fujian province(no1), capacity utilization in Fujian province(cu1), average inventory in Fujian province(it1) and employment in Fujian province(em1).

Table 7 list results for the quantile regression analysis of sub-indicators of LPI in Fujian province between column(2) and column(6). The quantile regression of LPI in Fujian province are performed at different quantiles, specifically at the quantile $q=0.10,0.25,0.50,0.75$ and 0.90 in Table 7. Here, we compare the results with one another and ordinary least square estimates. In the end, the estimates of sub-indicators of LPI in Fujian province between the column (2) and column(6) are statistically significantly different from zero at the $1 \%$ level, which contain business volume in Fujian province(bv1), new orders in Fujian province(no1), capacity utilization in Fujian province(cu1), average inventory in Fujian province(it1) and employment in Fujian province(em1), which is the same as the results of ordinary least square regression.

Our main results follow. First, business volume in Fujian province(bv1) increases gradually at the quantile $\mathrm{q}=0.50$, and decreases later, and finally reaches the maximum at the quantile $\mathrm{q}=0.90$. Second, new orders in Fujian province(no1) drops from 0.311 at the quantile $q=0.10$ to 0.302 at the quantile $\mathrm{q}=0.50$, then rises to 0.307 at the quantile $\mathrm{q}=0.75$, and finally it falls to 0.303 at the quantile $q=0.90$. Third, capacity utilization in Fujian province(cu1) reduces gradually, which is not the same as new orders in Fujian province(no1). Forth, average inventory in Fujian province (it1) rises at the quantile $\mathrm{q}=0.75$, which reaches 0.0992 , and reduce to 0.0938 at the quantile $\mathrm{q}=0.90$. Fifth, employment in Fujian province(em1) is also up to 0.209 at the quantile $q=0.75$, then falls to 0.208 at the quantile $\mathrm{q}=0.90$, so we reject the hypothesis 3 test. 


\section{Conclusion}

In conclusion, the data of LPI both in China and Fujian province are useful as a fore-indicator of economic and business conditions in China. From the analysis above, the results are listed below. First, the indexes of LPI in Fujian province are smaller than the indexes of LPI in China. Second, the indexes of LPI in Fujian province in April 2014 and April 2015 are larger than the indexes of LPI in Fujian province in May 2015 to November 2016. Third, the sub-indicators of the LPI in Fujian province do not increase over time.

The LPI in Fujian province has been strongly influenced by the policy, especially 21 st-Century Maritime Silk Road. So we make the following proposals. First, Fujian province should deep their cooperation with Taiwan, and promote the development of the Fujian Marine Economic Pilot Zone. Meanwhile, we should strengthen the port construction of coastal cities such as Fuzhou, Xiamen, Quanzhou and Zhangzhou.Second, business volume in Fujian province(bv1), new orders in Fujien province(no1), capacity utilization in Fujian province(cu1), average inventory in Fujian province(it1) and employment in Fujian province(em1) have significant effect on logistics prosperity index in Fujian province. So we should pay more attention to expand business volume, new orders, capacity utilization, average inventory and employment in Fujian province, but also we should improve capacity utilization and operating profit, and reduce logistics service charges and operating cost. on the other hand, we should accelerate inventory turnover and cash flow, At last, we should optimize business expectations in Fujian province.

\section{Reference}

[1] Marti L, Puertas R, García L. Relevance of trade facilitation in emerging countries' exports. The Journal of International Trade \& Economic Development. 2014,23(2):202-222.

[2] Martí L, Puertas R, García L. The importance of the Logistics Performance Index in international trade. Applied Economics. 2014,46(24):2982-2992.

[3] Puertas R, Martí L, García L. Logistics performance and export competitiveness: European experience. Empirica. 2014,41(3):467-480.

[4] YAN F, WANG Y. Analysis on Logistics Performance of the Silk Road Economic Zone Based on LPI. China Business and Market. 2016,8:005.

[5] FAN X, YU S. Empirical Analysis on Impact of Logistics Performance of Maritime Silk-Road Economic Belt on China'Import and Export Trade. Journal of Xi'an Jiaotong University (Social Sciences). 2015,3:004.

[6] xiaomei 1. The trend of China's LPI cycle decomposition and its economic implications. Journal of Commercial Economics 2016(11):75-77. 